\title{
Review of: "Gender effects on outcomes of psychosomatic rehabilitation are reduced"
}

\author{
Anna Panzeri
}

Potential competing interests: The author(s) declared that no potential competing interests exist.

The article is clear and well written, it addresses an interesting topic.

Future research may deepen the diffences among groups of different ages, with particular attention to the elderlies above 75 years old. Indeed, some studies suggested that physical outcomes may vary according also to the reported depression levels (Balestroni G. et al., 2020) and in different way for male and female gender, with worse outcomes for the latter (Panzeri, Komici, et al., 2021). Gender effects on physical a psychological rehabilitation is an intriguing topic still deserving further attention. 